Peter Auer (Freiburg i.Br.)

\title{
Die Geschichte der germanistischen Soziolinguistik in Deutschland: eine Skizze
}

\begin{abstract}
Der Beitrag versteht sich als erster Schritt zur historiographischen Rekonstruktion der Soziolinguistik in der Bundesrepublik Deutschland. Es wird gezeigt, wie in gewolltem Bruch mit der älteren germanistischen Forschung zum Thema Sprache und Gesellschaft in den späten 1960er Jahren die neue Disziplin der Soziolinguistik in Auseinandersetzung mit den Theorien Bernsteins entstand, sich die Soziolinguistik anschließend professionalisierte und das Spektrum ihrer Themen verbreiterte, schließlich auch den Anschluss an ältere Theorien insbesondere in der Dialektologie wiederfand.
\end{abstract}

\section{Einführende Bemerkungen}

Die Geschichte der Soziolinguistik in Deutschland umfassend darzustellen, bleibt ein Forschungsdesiderat. In dieser historiografischen Skizze kann lediglich versucht werden, die wichtigsten Entwicklungslinien nachzuzeichnen. Ich werde mich dabei weitgehend auf die Bundesrepublik beschränken, d.h. insbesondere die soziolinguistische Forschung in der DDR-Germanistik wegen ihrer grundlegend anderen politischen Einbettung sowie die germanistische Forschung in der Schweiz wegen der grundlegend anderen sprachlichen Bedingungen ausblenden.

Die Entwicklung der bundesdeutschen, germanistischen Soziolinguistik weist einige Besonderheiten auf, die sie z.B. von den englischsprachigen Ländern unterscheidet. Die wichtigste ist wohl, dass sie sich selbst als Erfindung der 1960er und 1970er Jahre verstand und inszenierte. (Dittmar 1973, S. 160, spricht von der „Genese einer neuen Wissenschaft“ in den späten 1960er Jahren.) Der Romanistin SchliebenLange ist dieser „Bruch mit der gesamten germanistischen Tradition“ (1973, S. 53) schon früh aufgefallen, wenn sie in ihrer Einführung in die Soziolinguistik schreibt:

[...] die deutsche Germanistik scheint hier gleichsam unter einem geradezu traumatischen Zwang zum Vatermord zu stehen. So wird vielfach mit der gesamten germanistischen Tradition gebrochen (Weisgerber; Dialektologie); und die kritiklose Rezeption von transformationeller Grammatik und Soziolinguistik Bernsteinscher Provenienz soll die Auseinandersetzung mit fünfzig Jahren Sprachwissenschaft ersetzen. Diese allgemein Traditions- und Orientierungslosigkeit der germanistischen Linguistik steht im Hintergrund der breiten Soziolinguistik-Rezeption. (Schlieben-Lange 1973, S. 54) 
Was die Soziolinguistik in Deutschland allerdings mit der im Ausland vereint, ist die Vagheit ihres Gegenstandsbereichs, die höchstens noch von der Pragmatik überboten wird. Sie umfasst heute so verschiedene Arbeitsgebiete wie die soziale Dialektologie, die Mehrsprachigkeitsforschung, die Variations-und-Wandel-Forschung, die anthropologische Linguistik etc. Man wird den Gegenstandsbereich der soziolinguistischen Forschung also eher im Sinn einer Wittgensteinschen Familienähnlichkeit verstehen müssen. Trotz aller Unbestimmtheit lassen sich aber drei zentrale Themenbereiche identifizieren und über die Jahrzehnte hinweg verfolgen:

\section{a) soziale Schichten oder Gruppen und ihre Sprache}

Es ist vielleicht die zentrale Aufgabe der Soziolinguistik zu untersuchen, wie sprachliche Formen auf soziologisch definierte Gruppen bezogen werden können. Diese gesellschaftlichen Gruppen können unterschiedlich groß und unterschiedlich abstrakt sein, von Klassen oder Schichten über Berufs-, Gender-, Altersgruppen bis zu Kasten, von sozialen Milieus bis zu Netzwerken und communities of practice. Was genau unter dem ,Bezug“ zwischen ihnen und der Sprache zu verstehen ist, ist eine der zentralen theoretischen Fragen der Soziolinguistik. Hier reicht das Spektrum von essentialistischen Modellen (die Frauen, Bauern, Arbeiter [...] sprechen so, wie sie sprechen, weil sie Frauen, Bauern, Arbeiter [...] sind') bis zu konstruktivistischen Modellen (,Frauen, Bauern, Arbeiter [...] werden überhaupt erst als soziale Kategorien erkennbar, weil sie sich in einer bestimmten Weise sprachlich verhalten und damit soziale Kategorisierungen auslösen'); manche Modelle postulieren oder implizieren einfache semiotische Beziehungen (,wat statt was bedeutet Arbeiterschicht'), andere arbeiten mit einer indexikalischen Semiotik, die einzelne linguistische Variablen mit einer Vielzahl sozialer Bedeutungen in einem „,indexikalischen Feld“ (Eckert 2008) assoziiert, die in verschiedenen Situationen und Kontexten evoziert werden können. Schließlich lässt sich die Beziehung zwischen sprachlichen Merkmalen oder Varietäten und sozialen Gruppen bzw. Kategorien direkt oder über vermittelnde Konstrukte wie das des (sozialen) Stils (Eckert 2004; Auer 2007) oder des Habitus (Bourdieu 1994 [1998]) modellieren.

Ein Teilbereich dieses Phänomenkomplexes ,Sprache gesellschaftlicher Gruppen', der aber wegen seiner außerordentlichen Bedeutung für die Soziolinguistik ab den Mitt-1960er Jahren besonders erwähnt werden muss, ist das Problem der Reproduktion sozialer Unterschiede im und durch das Bildungssystem, ein Problem, das auch heute wieder unter dem Stichwort soziale Selektion und Mobilität insbesondere in Bezug auf Schüler mit Migrationshintergrund eine bedeutende Rolle spielt. Die Soziolinguistik ist seit den späten 1960er Jahren sehr eng 
mit der Erziehungswissenschaft verbunden, und ihr Einfluss auf die Veränderung des deutschen Schulsystems in den letzten Jahrzehnten des letzten Jahrtausends sollte nicht unterschätzt werden.

\section{b) Sprachwandel}

Eine zunächst ganz andere soziolinguistische Thematik zielt auf die sozialen Bedingungen sprachlichen Wandels ab. Die enge Beziehung zwischen Variation und Wandel ist von Beginn der Sprachwissenschaft an gesehen worden, tragfähige Modelle für die Entstehung und Verbreitung neuer Formen in einer Gesellschaft sowie für die Verdrängung von Varietäten und Sprachen zugunsten anderer zu entwickeln, ist aber nach wie vor ein zentraler Gegenstand der Soziolinguistik. In Deutschland ist die Forschung zum Sprachwandel oft auch an die Frage der Etablierung und Ausbreitung der deutschen Standardvarietät und an Fragen des Dialektabbaus gebunden. Verbindungen bestehen zum Bereich a) insofern, als viele Sprachwandelmodelle soziale Schichten, Gender und Alter integrieren. Jedoch muss nicht jede soziale Verteilung einer Variablen schon Wandel implizieren.

\section{c) Sprachliche Minderheiten, Sprachpolitik, Weltsprachen}

Oft laufen diese Themen auch unter der (älteren) Bezeichnung Sprachsoziologie, weil sie nicht nur innerhalb der Linguistik, sondern auch in Politologie, Soziologie und Anthropologie bearbeitet werden. Die Diskussion um die so genannte Globalisierung hat diesen Bereich der Soziolinguistik in den letzten beiden Jahrzehnten wieder besonders aktuell gemacht.

Nur am Rand werde ich hier eine vierte Forschungstradition aus dem Themenbereich Sprache und Gesellschaft behandeln, die man unter das Stichwort Sprache als Weltsicht subsumieren könnte. Ihre eminente, für die Sprachwissenschaft im 19. und frühen 20. Jahrhundert bestimmende Rolle ist offensichtlich (vgl. die Diskussion um die Völkerpsychologie, aber auch die gesamte HumboldtRezeption bis zu Leo Weisgerber). In jüngerer Zeit wird die Weltsicht-Problematik kaum mehr als soziolinguistisches Problem diskutiert (sondern als eines der in Deutschland nur wenig etablierten linguistischen Anthropologie), sie spielt jedoch bis in die 1970er Jahre eine unterschwellige Rolle (siehe dazu unten).

Ebenso vernachlässige ich hier die Auseinandersetzung mit Sprache innerhalb der phänomenologischen Soziologie, die in Deutschland eng mit dem in den USA sozialisierten Thomas Luckmann und seinen Schülern (und über seinen in die USA emigrierten Lehrer Alfred Schütz auch indirekt mit der Ethnomethodologie und der von ihr ausgehenden soziologischen Konversationsanalyse) verbunden ist. Hier geht es um sprachliches Handeln, weniger um die Konstruktion 
sozialer Kategorien durch sprachliche Merkmale. Die Berührungspunkte zwischen interaktionaler Soziolinguistik und sprachsoziologischer Handlungstheorie bedürften einer eigenen historiografischen Rekonstruktion.

\section{Sprache und Gesellschaft in der Sprachwissenschaft vor 1965}

$\mathrm{Zu}$ keinem der im letzten Abschnitt genannten drei Themengebiete hat die Forschung erst in den 1960er Jahren begonnen, auch wenn der Begriff ,Soziolinguistik' erst aus dieser Zeit stammt. Es ist deshalb notwendig, sich zumindest vor Augen zu führen, auf welchem Stand der Forschung die Neuerfindung der Soziolinguistik in den 1960er Jahren stattfand.

\subsection{Sprachwandel}

Die wichtigste Forschung zu den sozialen Bedingungen sprachlicher Variation sowie sprachlichen Wandels fand in der ersten Hälfte des 20. Jahrhunderts in der Dialektologie statt. Diese hatte zu diesem Zweck um die Wende zum 20. Jahrhundert eine eigene, erfolgreiche, wenn auch indirekte Methodologie entwickelt, nämlich die so genannte kulturgeschichtliche Interpretation der Kartenbilder des Sprachatlasses des deutschen Reichs. Trotz aller Beschränkungen, die aus der selektiven Auswahl der Informanten und Phänomene für den Atlas resultierten, war die Karteninterpretation ein empirisch abgesichertes, erprobtes und nachprüfbares Verfahren. Und im Gegensatz zur heutigen (traditionellen) Dialektologie, deren Arbeit sich oft in der Dokumentation und Archivierung grunddialektaler Daten erschöpft, wurde das Instrument auch vielfach genützt, um Generalisierungen zum Sprachwandel aufzustellen. Um lediglich ein Beispiel zu nennen: Der ,Vorbruch anhand einer Kommunikationsader' als Kartenbild für die aus Norden kommenden Innovationen im Alemannischen entlang des Rheins ist zunächst eine empirische Feststellung über die geografische Verbreitung bestimmter sprachlicher Merkmale; diese muss jedoch sozio-kulturell erklärt werden, insbesondere weil der ,Vorbruch“ linksrheinisch (also im Elsass) deutlich weiter in den Süden reicht als rechtsrheinisch. Die Erklärung setzt ein wie auch immer geartetes, komplexes Modell des ,Transports‘ der Sprache zusammen mit den Waren oder Menschen auf dem Handelsweg Rhein voraus, das neben wirtschaftlichen Beziehungen auch politische Machtverhältnisse und kulturelles Prestige integriert. 
Adolf Bachs maßgebliches Überblickswerk „Deutsche Mundartforschung“ von 1950, eine stark überarbeitete Version seines Buchs von 1934, fasst zahlreiche Forschungsergebnisse wie auch theoretische Konzepte aus der Vorkriegsdialektologie zusammen, die teils spätere sozio-dialektologische Theorien vorwegnehmen. Dazu gehört das Phänomen des (von Chambers/Trudgill 1980, S. 196-202 später so genannten) city-hopping, das schon im 19. Jahrhundert wohl bekannt war (vgl. Bach 1950, S. 135 f.) und zum Beispiel als Modell für die Verbreitung der uvularen R-Variante verwendet wurde: Neuerungen erobern zunächst städtische Gemeinschaften, die am ehesten anfällig für das Prestige dieser Formen sind, um von dort in die umgebende Landschaft einzudringen; dort stellt sich ihnen allerdings manchmal ein ,vom Leben der Stadt unabhängige[s], selbstbewußte[s] Bauerntum“ entgegen, das dieses Vordringen verhindert, so dass die Städte als Inseln im Kartenbild erscheinen (ebd., S. 135; eine moderne Version davon findet sich z.B. bei Vanderkerckhove 2010).

Die Diskussion der Vorkriegszeit kreiste um die Frage, welche Erklärungen für Kartenbilder angemessen sind, die sprachliche Wandelphänomene nahe legen. Zur Disposition stand die junggrammatische Auffassung, alle Veränderung im Raum könne allein aus der Stärke des Kommunikationskontakts (also, in modernen Begriffen, aus der Netzwerkdichte und -stärke) erklärt werden. Dagegen setzte sich in der deutschen Dialektologie der Vorkriegszeit immer mehr die Auffassung durch, dass „Verkehr“ allein den horizontalen Wandel nicht erklären kann; insbesondere Walther Mitzka (etwa in Mitzka 1928) plädierte dafür, dass sich Sprachlandschaften verschieben, ,indem das Machtgefühl der einen Seite im wirklichen Sinne des Wortes Eindruck macht“ (S. 61), diese Seite also einen „Mehrwert der Sprachgeltung“ entwickelt - eine Auffassung, der auch Bach folgt. Die Auseinandersetzung zwischen positivistischen, auf Kontaktfrequenz aufbauenden, und sozio-interpretativen, auf Einstellungen und Bewertungen operierenden Ansätzen bei der Erklärung des sprachlichen Wandels ist bis heute nicht beendet (vgl. Auer/Hinskens 2005).

\subsection{Sprachliche Gruppen/Schichten und ihre Sprache}

Bach widmet der Beziehung zwischen sozialen Gruppen und sprachlichen Merkmalen ein ganzes Kapitel seines Buchs (Kap. V: „Mundart in ihrer soziologischen Schichtung“). Es diskutiert ausführlich den Einfluss von Schichten, aber auch von Alter, Stadt vs. Land, Industrialisierung und Berufsgruppen (Zuzug von Industriearbeitern).

Ein zentraler Bezugspunkt der Diskussion in der Vorkriegszeit zum Thema Sprache und soziale Schicht sind die Arbeiten des Altgermanisten und Volks- 
kundlers Hans Naumann, dessen in den 1920er Jahren entwickelte Theorie des „gesunkenen Kulturguts“ eine beträchtliche Wirkung entfaltete (vgl. Naumann 1922, 1925). Dass Naumann - wie nicht wenige Mediävisten, Dialektologen, Nordisten und Volkskundler - ab 1933 zum fanatischen Nationalsozialisten wurde, ${ }^{1}$ was ihn 1945 sogar zeitweise seinen Bonner Lehrstuhl kostete, ist sicherlich ein Teil der Erklärung dafür, dass er in der neuen Soziolinguistik völlig aus dem kollektiven Gedächtnis getilgt wurde. Naumanns von Levy-Brühl angeregte Überlegungen zum „sprachlichen Verhältnis von Ober- zu Unterschicht“ (so der Titel eines Aufsatzes von 1925) sind aber nicht nur von großer Bedeutung für das Denken seiner Zeit; sie nehmen auch wesentliche Aspekte der Labovianischen Unterscheidung zwischen change from above und change from below vorweg. Im Gegensatz zu den Junggrammatikern, die insbesondere den Lautwandel ausschließlich als change from below sahen, betonte Naumann das Nebeneinander beider Richtungen des Sprachwandels. Change from above bedeutet für ihn den „Einfluss der Kultursprache auf die Mundart“ (,gesunkene Kultur“); auf diesem Weg seien Innovationen vor allem „durch die Berührung der heimischen [bilingualen] kulturellen Oberschicht mit fremdstämmigen Kulturkomplexen, besonders an der Peripherie des Stammestums in die deutsche Kultursprache und von da aus alsdann in die deutschen Mundarten gedrungen“ (1925, S. 57). Die Innovationen der Oberschicht seien „Moden“, die gerade zur distinktiven Abgrenzung von den unteren Schichten dienten, sie folgten also keineswegs dem junggrammatischen Muster endogenen Wandels. Dagegen setzt er den change from below: Hier läuft Naumanns Theorie letztendlich auf die Generalisierung hinaus, dass Lenisierungen (phonologische Abschwächungen aller Art, auch zu Lasten der Morphologie) sich nur in den ,unkontrollierten` Unterschichtsidiomen ausbreiten können.

Stark beeinflusst von Naumanns Betrachtungen ordnen sich entsprechend auch bei Bach (1950)

die „Schrift- und Gemeinsprache“ (zumindest in bestimmten Situationen) der „Bildungschicht“, sonst der Bühne,

die „landschaftliche Umgangssprache“ (heute würde man von Regionalstandard sprechen) der „Bildungsschicht“ und „Mittleren Schicht“,

die „Halbmundart“ (heute: Regionaldialekt) der „Mittleren Schicht“ und als nicht erreichte Idealsprache der „Grundschicht“, sowie schließlich

die Mundart der „Grundschicht“

1 Auch Adolf Bach und Walter Mitzka waren zum Beispiel NSDAP-Mitglieder seit 1933. 
zu. Dieses sprachlich-soziale Schichtungsmodell ermöglicht es ihm, Hyperkorrekturen im Sinne von Hyperstandardformen und Hyperdialektalismen (ebd., S. 232) zu diskutieren.

Die Dialektologie der Vorkriegszeit war also in hohem Maß an den sprachlichen Korrelaten sozialer Schichtung bzw. Gruppenbildung interessiert und wusste dieses Interesse auch auf die Erklärung des Sprachwandels anzuwenden. Spätestens in der Nachkriegszeit waren allerdings die Möglichkeiten des methodischen Instruments ,Karteninterpretation‘ ausgeschöpft. Während das Verfahren am Beginn des Jahrhunderts innovativ und auch für andere Sozialwissenschaften beispielgebend war, kam es nun zur Stagnation. Die inzwischen reichlich vorhandenen und hoch entwickelten Methoden der empirischen Sozialforschung wurden nicht genutzt, die Arbeit mit Corpora und die Anwendung statistischer Verfahren waren völlig unbekannt. Die Dialektologie beschränkte sich immer mehr auf eine archivarische Rolle und geriet ins Abseits der sprachwissenschaftlichen Diskussion.

Neben der Dialektologie gab es in der ersten Hälfte des 20. Jahrhunderts noch einen zweiten großen Arbeitsbereich der germanistischen Soziolinguistik (ante litteram), der ebenfalls ins 19. Jahrhundert zurückreicht, nämlich die historische Forschung zur Entstehung und Verbreitung der deutschen Gemein- bzw. Standardsprache. Hier war das Datenmaterial anders als in der Dialektologie nicht die mündliche Volkssprache, sondern die schriftlichen Dokumente mehr oder weniger gebildeter Schreiber. Fragen der sozialen Differenzierung springen aber sofort ins Auge, wenn man zum Beispiel den Übergang einer niederdeutschen Stadt zum Hochdeutschen analysiert, das ja über die oberen Schichten importiert wurde (durchaus in Naumanns Sinn). Die Arbeiten der historischen Stadtsprachenforschung - stellvertretend seien die immer noch höchst lesenswerten Arbeiten Agathe Laschs zum Berlinischen genannt (Lasch 1910 und 1928) - sind deshalb im Grund immer auch soziolinguistische Arbeiten.

\subsection{Sprachpolitik und Minderheitenfrage}

Der einflussreichste Beitrag der älteren deutschen Forschung zur internationalen Soziolinguistik kam jedoch nicht von Dialektologen, Sprachgeschichtlern oder Volkskundlern, sondern stammt aus dem dritten Themenbereich der Soziolinguistik (oder Sprachsoziologie), nämlich der Sprachpolitik und Minderheitenforschung. Ich meine Heinz Kloss’ Beiträge zu diesem Thema, von den späten 1920er Jahren (1929: „Nebensprachen“) bis in die späten 1960er Jahre (1969: „Grundfragen der Ethnopolitik im 20. Jahrhundert“). Gerade Kloss’ theoretisch-konzeptuelle Beiträge wurden - früher noch als in Deutschland - in der nordamerika- 
nischen Linguistik weithin rezipiert und geschätzt (nicht zuletzt, weil Kloss auch auf Englisch publizierte). Unterscheidungen wie die zwischen Abstand- und Ausbausprachen, Kloss’ Ausführungen zu plurizentrischen Sprachen sowie sein Konzept der „dachlosen Mundart“ gehören zum Kern soziolinguistischer Begrifflichkeiten und sind in einer Weise linguistisches Allgemeingut geworden, die wohl kein anderer deutscher Linguist des 20. Jahrhunderts für sich beanspruchen kann.

Heute ist Kloss' Bedeutung für die Soziolinguistik unbestritten. Nicht selten wird dabei allerdings seine frühe Karriere vergessen: Kloss war seit 1927 Mitarbeiter und später Abteilungsleiter im Deutschen Auslandsinstitut in Stuttgart, das die Aufgabe hatte, die Auslandsdeutschen für das Dritte Reich zu gewinnen. Er war dort 1938 bis 1945 u.a. als Leiter der „Arbeitsstelle für Volksforschung“ direkt an der Ausarbeitung der Volkstumsideologie der Nationalsozialisten beteiligt. Kloss war aber vor allem ein Technokrat; seine Aufgabe war es, statistische und andere Informationen über die Deutschen im Ausland $\mathrm{zu}$ sammeln und daraus politische Empfehlungen zu entwickeln. Zu seinem internationalen Ruhm in der Nachkriegszeit und der Wertschätzung, die er gerade bei Soziolinguisten wie Joshua Fishman genoss, dürfte der sachliche, scharfsinnige, oft terminologisch um feine Differenzierungen bemühte Stil seiner Publikationen sowie sein enormes Wissen über die Minderheiten überall in der Welt beigetragen haben. Dass die Reise eigentlich woanders hin gehen sollte, zeigen jedoch einige seiner Kriegspublikationen, z.B. die populäre nationalsozialistische Propagandaschrift „Brüder vor den Toren des Reiches“ (1941), in der er den „weltdeutschen“ Gedanken entwickelt und die Auslands- und Volksdeutschen im Rahmen des Schicksals eines „Großvolkes, dem es versagt war, eine politische Großmacht zu bleiben“ (ebd., S. 17), diskutiert; diese Spannung habe erst das Dritte Reich und der Krieg aufgelöst. ${ }^{2}$ Kloss vergleicht die beiden „Großvölker“ der Engländer und Deutschen. Der fast obsessive Gedanke des Heftchens ist, dass nur die Engländer den einem „Großvolk“ auch angemessenen Status einer politisch-militärischen Großmacht erreicht hätten, bei dem Sprach- und Machtbereich übereinstimmten, während gerade die Oberschicht der deutschen Emigranten sich sprachlich assimiliert hätte und damit zwar rassisch, aber nicht mehr sprachlich deutsch sei. Ganz im Sinne der nationalsozialistischen Rassenlehre steht Kloss in einer anti-humboldtianischen Tradition. Das Blut, nicht die Sprache definiert die Volkszugehörigkeit. Der Begriff der „dachlosen Minderheiten“ gewinnt in diesem Kontext ihren eigent-

2 Eine Monographie von 1944 mit dem Titel „Von Auftrag und Ordnung der Völker“ wurde nicht mehr veröffentlicht, lediglich das Inhaltsverzeichnis ist bekannt; vgl. http://homepages.unituebingen.de/gerd.simon/kloss.pdf. 
lichen Stellenwert: Er bezieht sich auf die Analyse der sprachlichen Defizite des Deutschtums im Ausland, das, so Kloss, durch verschiedene Mittel (auch die „Rückführung ins Reich“) bekämpft werden müsse. ${ }^{3}$

Das sprachsoziologische Interesse für die deutsche Sprache im Ausland teilte Kloss mit einigen anderen, für die soziolinguistische Theoriebildung aber langfristig irrelevanten Kollegen, insbesondere mit Franz Thierfelder, bis 1937 Präsident der Akademie zur Wissenschaftlichen Erforschung und Pflege des Deutschtums in München, der nach dem Krieg an der Umwandlung dieser Akademie in das Goethe-Institut beteiligt war. Seine statistisch orientierten Werke wie „Deutsch als Weltsprache“ (1938) gingen politisch in dieselbe Richtung wie die Kloss’, mit dem er in engem Austausch stand.

Kloss' Einfluss auf die amerikanische Soziolinguistik war schon in den 1960er Jahren bedeutend, während er in Deutschland in der neuen Soziolinguistik nicht zur Kenntnis genommen wurde. Das lag wohl weniger an seiner politischen Vergangenheit, die nur wenigen aus der jungen Generation der Soziolinguisten bekannt gewesen sein dürfte, als an deren generellem Desinteresse für Fragen der (deutschen) Minderheiten im Ausland sowie der geringen Relevanz der Minderheitenfrage für die Bundesrepublik selbst. Die Beschäftigung mit deutschen Sprachinseln oder deutschen Sprachminderheiten hatte den Hautgout der Vertriebenenverbände, die politisch zum äußersten rechten Rand der BRD gehörten und für die meist am linken Rand operierenden Soziolinguisten der 1968er-Generation ,Revisionisten“ waren. Das dürfte übrigens auch der Grund dafür sein, dass die soziolinguistisch hoch interessante Problematik der Ost-Vertriebenen und -Flüchtlinge in der bundesdeutschen (und auch DDR-) Soziolinguistik weitgehend unbearbeitet blieb. Migration war in dieser Zeit grundsätzlich noch kein soziolinguistisches Thema.

\section{Die neue Soziolinguistik 1965-1980}

\subsection{Sprache und Reproduktion sozialer Ungleichheit in der/durch die Schule}

Die neue Soziolinguistik startet in den späten 1960er Jahren ohne jeden expliziten Bezug auf die im letzten Abschnitt skizzierten Traditionslinien. Ihre allererste Phase bis ca. 1970 ist vielmehr fast vollständig durch die Rezeption der Arbeiten

3 Vgl. zu Kloss' Rolle im Dritten Reich die ausführliche Darstellung bei Hutton (1999, Kap. 5). 
des britischen Sozialpsychologen Bernstein (u.a. Bernstein 1964) bestimmt. Indirekt ist allerdings zumindest eine Form der Kontinuität von der älteren zur neueren Soziolinguistik zu erkennen. Sie läuft über das Thema ,Sprache als Weltsicht‘, das z.B. in Dittmars einflussreicher, in vielen Tausend Exemplaren verkaufter Einführung (erstmals 1973) noch als Teil der Soziolinguistik behandelt wird. Bernsteins Vorstellung, der restringierte Code der Arbeiterkinder bestimme ihre (defizitäre) Denkweise, lässt sich nämlich durchaus als Variante der Humboldtschen Auffassung der Prägung des Denkens durch die Sprache sehen, das hier nun von Volk oder Nation auf die soziale Klasse als Referenzpunkt umgewendet wird. Und nicht einmal diese Wendung war ganz unbekannt, denn in der älteren deutschen Sozialdialektologie (z.B. bei Bach) geht es ja auch um die „Weltsicht des Dialekts“, die als eine bestimmte Denkweise verstanden wird, die durch die sprachlichen Strukturen bedingt ist. ${ }^{4}$

Bernsteins Idee war, dass Kindern der Arbeiterschicht der soziale Aufstieg verwehrt wird, weil sie ihre Sprache trotz guter non-verbaler Intelligenz daran hindert, die Denkstrategien zu entwickeln, die die von der Mittelschicht geprägte Schule fordert. Bernsteins Schriften kombinieren also eine psycholinguistische mit einer soziologischen und diese mit einer pädagogischen Sichtweise und entwickeln ein recht komplexes Modell für den Zusammenhang von milieutypischen sprachlichen Interaktionsformen, grammatischen Kompetenzen, Kognition und Schichtenzugehörigkeit.

Der einflussreichste Adept dieser Theorie in Deutschland war der Soziologe Oevermann, dessen 1967 eingereichte Frankfurter Dissertation laut Vorwort ihren Ursprung ,in einer längeren Diskussion mit Basil Bernstein im Winter 1964/65“ (1970, S. 9) hatte. Das enorme Echo, das Bernstein und Oevermann in der deutschen Linguistik und Erziehungswissenschaft fanden, lässt sich auch daran erkennen, dass Oevermanns Dissertation 1970 nur wenig verändert unter dem Titel „Sprache und soziale Herkunft - Ein Beitrag zur Analyse schichtenspezifischer Sozialisationsprozesse und ihrer Bedeutung für den Schulerfolg“ auflagenstark als stw-Band erschien, obwohl die kleingedruckten 550 Seiten des Buchs keine leicht verdauliche Lektüre sind. Es wird darin viel theoretisiert und eine ermüdend genaue quantitative Untersuchung von Schüleraufsätzen aus einer einzigen (!) Frankfurter Realschulklasse vorgestellt. In Bernsteinscher Denkweise wird postuliert, dass Unterschichtskinder aufgrund des in ihrem Milieu herrschenden Sozialisationsstils sprachlich verarmt aufwachsen (und deshalb auch in verbalen IQ-Tests schlechtere Leistungen zeigen). Diese sprachliche Verarmung führe zu kognitiven Defiziten: „Der Grad der Sprachbeherrschung stellt nicht nur ein Persönlichkeits-

4 Auch dieser Diskurs reicht natürlich ins 19. Jahrhundert zurück. 
merkmal unter anderen dar, sondern ist relevant für die Gesamtheit der psychischen Funktionen“ (ebd., S. 38). Wenig abstrakte, kontextdeterminierte Begriffe und eine undifferenzierte Syntax störten die Entwicklung der Schüler. „Das Individuum ,übernimmt‘ also im Akt des Sprechens und im Prozeß des Spracherwerbs den sozial vorgegebenen Erfahrungshorizont seiner Umgebung“ (ebd., S. 77).

Die theoretischen und methodischen Probleme der Oevermannschen Studie waren evident und wurden schon bald (teils bereits von ihm selbst, der seiner Studie lediglich Pilotcharakter zugestand) erkannt. Innerhalb kürzester Zeit waren sie so vollständig Teil des common sense, dass Bernsteins Theorie nicht mehr ernsthaft diskutiert werden durfte.

Oevermanns methodische Probleme waren teils von Bernstein ererbt. Insbesondere waren in der Modellierung

\section{sprachliche Kodes $\leftrightarrow$ kognitive Stile $\leftrightarrow$ Schichten}

die kognitiven Stile ja eine black box: Der Zusammenhang zwischen ihnen und bestimmten linguistischen Merkmalen (bei Oevermann wurden - eher wahllos nicht weniger als 89 davon untersucht) war lediglich eine unbewiesene Annahme. Teilweise schuf sich Oevermann aber auch eigene Probleme. So versuchte er, Bernsteins Annahmen, die sich auf die mündliche Sprache bezogen, anhand schriftlicher Texte zu überprüfen. Bernsteins Überlegungen zur Kontextabhängigkeit bestimmter sprachlicher Strategien, so einleuchtend sie sein mögen, konnten in diesen schriftlichen Texten aber nicht mehr funktionieren. Ein zweites, vielleicht noch schwerwiegenderes Problem war es, in der untersuchten Realschulklasse überhaupt eine valide Unterscheidung zwischen US, UMS, MMS und OMS zu treffen; die soziale Hierarchie war in der Klasse - der Natur des dreigliedrigen deutschen Bildungssystems entsprechend - eher flach. Nur für wenige linguistische Merkmale waren daher die Schichtenunterschiede überzufällig. Allerdings konnte Oevermann ziemlich gut nachweisen, dass Mädchen mehr den elaborierten Kode verwenden als Jungen (ebd., S. 516), ein Ergebnis, das angesichts des dominanten Interesses an sozialer Schichtung und des Desinteresses an Gender-Unterschieden zu dieser Zeit aber kaum beachtet wurde.

Neben diesen methodischen und empirischen Problemen ergab sich für die Vertreter der linken Studenten- und Assistentenschaft durch die Bernsteinsche und Oevermannsche Argumentation auch ein politisches Dilemma: Die Theorie zwang sie nämlich, die Sprache der Arbeiterschicht als defizitär (,restringierter Kode“) einzustufen, was schon bald auf vehemente Ablehnung stieß. Das Pendel schlug nun in die umgekehrte Richtung aus und führte - in Schlieben-Langes Formulierung - zur „folkoristische[n] Glorifizierung der Unterschichtssprache; um der Erhaltung der Solidarität der Arbeitsklasse willen verzichtete man auf die Frage nach emanzipatorisch-reflexiven Möglichkeiten“ (1973, S. 57). 
Schon Anfang der 1970er Jahre wurden Konsequenzen aus der Kritik des Bernstein-Paradigmas gezogen.

Eine naheliegende Konsequenz war es, die black box der kognitiven Stile aus dem Modell zu eliminieren und die Reproduktion der sozialen Schichtenstruktur direkt mit der negativen Bewertung der sprachlichen Merkmale der Unterschicht durch die Lehrer und Lehrerinnen zu erklären. Dadurch konnte man auf die Charakterisierung der Unterschichtsprache als „restringiert“ verzichten. Die Reproduktion der sozialen Ungleichheit ergab sich schlicht aus der Normwidrigkeit dieser Sprache. Diese Argumentationslinie verfolgte z.B. Ammon in seiner Tübinger Dissertation von 1972 über „Dialekt, soziale Ungleichheit und Schule“ (vgl. Ammon 1973a und b), deren empirischer Teil sich allerdings auf den sehr differenzierten Nachweis von sozialen Unterschieden in der Erwachsenensprache beschränkt. Er untersuchte in Schwaben anhand der im Rahmen der so genannten ZwirnerErhebung von Ruoff gesammelten Daten die mündliche Sprache verschiedener Schichten in Interviews und fand wie Oevermann - erneut wenig beachtete Gender-Unterschiede (berufstätige Frauen sprechen standardnäher), konnte aber auch soziale und Alters-Unterschiede nachweisen.

Dieselbe argumentative Verschiebung lässt sich auch in Jägers Untersuchung zu den Normabweichungen in Diktaten und Aufsätzen von 450 Schülern im 4./6./9. Schuljahr in Mannheimer Grund- und Hauptschulen ${ }^{5}$ erkennen (1971). ${ }^{6}$ Jäger untersuchte ebenfalls eine Vielzahl orthografischer, grammatischer (teils dialektaler) und stilistischer Normabweichungen und stellt v.a. in der 9. Klasse mehr oder weniger deutliche Unterschiede in den orthografischen Kenntnissen fest; in den unteren Klassen fanden sich eher Unterschiede, die auf dialektale Interferenzen hindeuteten. Die Konsequenzen, die er aus seiner Untersuchung zog, zielten auf eine Veränderung des schulischen Bewertungssystems hin: Die „Erlernung des form- und sinnrichtigen Gebrauchs der Hochsprache“ wird als Bildungsziel abgelehnt, weil dadurch „,der Blick vom Wert der Eigensprache des Kindes abgelenkt“" werde (Jäger 1971, S. 166). Die normativen Kategorien ,falsch“ und ,richtig“ müssten zugunsten kommunikativer „Akzeptanz“ aufgegeben werden (ebd., S. 169). Kompensatorische Maßnahmen (also die besondere Förderung von Schülern und Schülerinnen aus bildungsfernen Elternhäusern) werden ab-

5 Das heute unvorstellbare Faktum, dass es zu dieser Zeit offenbar noch möglich war, Mittelschichtskinder in einer Hauptschule zu finden, lässt die zwischenzeitliche Wandlung des deutschen Schulsystems deutlich werden. Allerdings operationalisierte Jäger Schichtenzugehörigkeit über das Wohnviertel; es ist also möglich, dass die Hauptschulkinder aus den ,besseren` Vierteln tatsächlich gar nicht zur Mittelschicht gehörten, sondern quasi nur im falschen Viertel lebten.

6 Vgl. zu seinem Projekt „Schichtenspezifischer Sprachgebrauch von Schülern“ auch Jäger/ Feldbusch (1973). 
gelehnt. Verstöße gegen orthografische Regeln und die der Zeichensetzung sollten nicht mehr sanktioniert und die Beschäftigung mit diesem Thema ,auf das Notwendigste“ eingeschränkt werden (ebd., S. 228). Man wird zugestehen, dass dieses Ziel im deutschen Schulsystem, vor allem in den langzeitig SPD-regierten Ländern, sehr weitgehend erreicht worden ist (vgl. Steinig et al. 2009).

Eine sehr differenzierte Untersuchung legte Steinig in seiner Bonner Dissertation vor (Steinig 1976), diesmal anhand mündlicher Schülersprache im Ruhrgebiet (Filmnacherzählungen). Er führte umfangreiche Analysen zur Einstellung von (angehenden) Lehrern anhand von Sprachprobenbewertungen (Zuordnung der Stimmen zu Schultypen) und unter Anwendung des semantischen Differentials durch. Es zeigte sich unter anderem, dass PH-Studentinnen die Schicht der erzählenden Kinder sehr gut einschätzen konnten, und zwar unabhängig von deren IQ; dies deutet darauf hin, dass Kinder, die ,falsch“ sprachen, eben deshalb von den weiterführenden Schulen ferngehalten wurden, die Sprache also als soziale Barriere fungierte. Im Gegensatz zu anderen Studien ist Steinigs Untersuchung auf saliente, negativ bewertete Merkmale des Ruhrdeutschen beschränkt (Schwerpunkt sind Kasusverwechslungen und die dat/das-Alternation). In Auseinandersetzung mit Labov differenziert Steinig verschiedene Stile mit unterschiedlichen Interaktionspartnern. Er fand durchgängig einen starken Zusammenhang zwischen den stigmatisierten Ruhrdeutschformen und sozialen Indikatoren wie Beruf des Vaters und Bildungsaspirationen der Eltern für ihr Kind. Viel schlechtere Korrelationen ergaben sich hingegen bei Merkmalen, die Bernstein als Indikatoren des restricted code sah, etwa der Verwendung exophorischer Mittel (,egozentrisches Sprechen').

Der Boom des Themas Ungleichheit, Sprache und Schule in den späten 1960er und 1970er Jahren hatte nicht nur mit der politisch motivierten Kritik der 68er-Generation am sozialen Selektionsinstrument Schule zu tun. Vielmehr war in der Bundesrepublik kurz vorher von dem Philosophen Picht die „Bildungskatastrophe“ bzw. der „Bildungsnotstand“ ausgerufen worden (Picht 1964). Der weitere wirtschaftliche Aufschwung des Landes drohte am Fehlen qualifizierter Arbeitskräfte zu scheitern. Es mussten also qualifizierte Arbeitskräfte aus den unteren Schichten rekrutiert werden. Die Schulen sollten sich für diese Schichten öffnen. Die Frage, ob dazu auch die Bildungsstandards (Bildungspläne) geändert werden mussten, war von unmittelbarer praktischer Relevanz. Die Alternative war, entweder die Kinder aus bisher bildungsfernen Schichten durch besondere Fördermaßnahmen zu unterstützen oder die Verpflichtung der Schulen auf die strengen orthografischen, orthoepischen, grammatischen und stilistischen Normen der 1960er Jahre aufzugeben. Dittmar hat in seiner Einführung von 1973 diesen beiden Alternativen die Begriffe „Defizithypothese“ und „Differenzhypothese“ zugeordnet und erstere mit Bernstein, letztere mit Labov und 
seinem Kampf für die ,Gleichberechtigung' des afro-amerikanischen Englisch assoziiert. Er trieb damit die Rezeption der Labovianischen Soziolinguistik in der BRD voran, die mehr als die an Mittelschichtsnormen orientierte Theorie Bernsteins dem sich verbreitenden Sprachliberalismus entsprach - und dies, obwohl Dittmar im Vorwort konstatiert, dass zwischen Defizit- und Differenzhypothese „trotz erheblich verbesserten und verfeinerten wissenschaftlichen Methoden insofern kein großer inhaltlicher Unterschied besteht, als beide Konzeptionen in ihrem Anspruch, gesellschaftliche Ungleichheit aufzuheben, zu kurz greifen“ (Dittmar 1973, S. XI).

Dittmars Einführung (seiner Konstanzer Dissertation) kommt der Verdienst zu, die amerikanische Soziolinguistik in Deutschland populär gemacht zu haben. ${ }^{7}$ Neben Labov, Bickerton, DeCamp werden auch anthropologische Ansätze wie etwa die Ethnographie der Kommunikation ausführlich dargestellt, darunter die berühmte Hemnesberget-Studie von Blom/Gumperz (1972); auch Mehrsprachigkeit kommt vor. Entsprechend dem Anspruch des Buchs, zu einer Theoretisierung der (Sozio-)Linguistik beizutragen (was in der Zeit fast mit formalen Regeln identisch war), widmet sich Dittmar ausführlich technischen Darstellungsweisen wie der Labovschen Variablenregel. Die linguistischen Analysen wurden deutlich anspruchsvoller, die Einbettung in die großen sozialen Fragen der Zeit tritt aber bereits etwas zurück.

\subsection{Sprach- und Sozialstruktur: das größere Bild}

In den ersten Anfängen der neuen bundesdeutschen Soziolinguistik wurde die generative Transformationsgrammatik (wie sie damals hieß) noch nicht als Gegenpol zur Soziolinguistik empfunden. Zahlreiche Verweise auf Chomsky in den soziolinguistischen Publikationen dieser Zeit belegen, dass viele Grundannahmen der generativen Grammatik auch aus soziolinguistischer Perspektive attraktiv erschienen; außerdem verband beide Strömungen die Ablehnung der ,traditionellen' germanistischen Sprachwissenschaft. Im Laufe der 1970er Jahre entwickelten sich die zunehmend formal werdende, an Montague und Chomsky orientierte ,Systemlinguistik' und die Soziolinguistik/Pragmatik jedoch auseinander, und „unterschwellige Spannungen“ traten auf (so Becker/Dittmar/Klein 1978,

7 Neben seiner Einführung sind auch die Sammelbände von Klein/Wunderlich (Hg.) (1971) und der Übersetzungsband mit Labovs Schriften (Labov 1976/1978) zu nennen, die ebenfalls zur Rezeption der Labovianischen Soziolinguistik beitrugen. 
S. 159), die sich durchaus polemisch manifestierten. So formulierte Klein seine grundlegende Skepsis gegenüber einer Soziolinguistik, die Sprach- und Sozialstruktur aufeinander beziehen will, wie folgt:

Ob der transformationelle Zyklus so oder so läuft [...] steht in keinerlei Zusammenhang mit den Einkommensverteilung, der Rolle der Frauenverbände, dem Hauptwiderspruch und was immer man für eine Sozialstruktur als wichtig erachten mag. (Becker/Dittmar/ Klein 1978, S. 161)

Hier deutet sich an, was wohl noch heute gilt, nämlich dass die sprachlichen Phänomene, für die sich formale Grammatiktheorien interessieren, kaum eine Überschneidungsmenge mit jenen haben, die für soziale Unterschiede sensitiv sind.

Aber auch innerhalb der Soziolinguistik lässt sich in dieser Zeit eine Art Arbeitsteilung beobachten. Während auf der einen Seite die technische Beschreibung der sprachlichen Variation immer anspruchsvoller wurde, beschäftigte sich eine andere Richtung der Soziolinguistik mit avancierteren Modellen zur Erfassung der sozialen Bedeutung sprachlicher Variation.

Die Fortschritte der Labovianischen Variationslinguistik gegenüber der linguistisch naiven Bernstein-Soziolinguistik waren offensichtlich. Allerdings hat das Beschreibungsinstrument der Variablenregel in der bundesdeutschen Linguistik meines Wissens nur eine überzeugende Anwendung gefunden: nämlich in Schlobinskis Berlin-Studie, in der die Schwächung von silbenanlautend /g/ als Variablenregel formuliert wird - aber das war schon weit in den 1980er Jahren (1987). Eine germanistische Sonderentwicklung ist nämlich auch darin zu sehen, dass in Deutschland anders als im englischsprachigen Raum sprachliche Variation gern nicht als Variation innerhalb einer Varietät modelliert wurde (wie dies bei Labov geschieht), sondern als Komplex von Varietäten innerhalb einer Sprache - daher die in Deutschland weit verbreitete Bezeichnung „Varietäten-“ (statt „Variations-")Linguistik. Ohne Zweifel ist die deutsche (germanistische und romanistische) Sichtweise stark von Coserius „Architektur“ einer historischen Sprache geprägt, die er schon in den späten 1950er Jahren entwickelt hatte (1955). Coseriu dehnte den Varietätenbegriff der Dialektologie (diatopische Dimension) vom geografischen Raum auf soziale Gruppen und Stile aus (diastratische und diaphasische Dimension): ${ }^{8}$ Alles was ein Sprecher einer bestimmten geografischen Herkunft aus einer bestimmten sozialen Gruppe in einer bestimmten Situation äußert,

8 In dieser Tradition steht z.B. die Einführung Löfflers in die Soziolinguistik (2005, S. 79); vgl. ebenso den entsprechenden Eintrag in Bußmann (Hg.) (2002, S. 730). 
ist demnach eine Varietät. Da solche Varietäten nicht mehr diskret sein können, kommt man fast zwangsläufig zu einer probabilistischen Auffassung, wie sie Klein 1974 in seiner „Varietätengrammatik“ formulierte. Er sieht eine solche Grammatik als Abbildung eines Produkts aus einer Menge von zeitlichen Perioden (er schließt auch historische Variation mit ein), einer Menge von geografischen Räumen, einer Menge von Schichten und einer Menge von Situationstypen auf eine Menge von probabilistisch bewerteten grammatischen Regeln (Klein 1974, S. 59).

Für Klein sollte diese probabilistische Grammatik alle Erscheinungsformen des Deutschen, also (anders als bei Coseriu) auch die historischen, abdecken. Für jede Kombination von Sprechertyp, Sprecherherkunft, Situation und historischer Sprachstufe würde jeder Regel eine Wahrscheinlichkeit ihrer Anwendung zugeordnet, bei nicht mehr gültigen Regeln die Wahrscheinlichkeit Null. Die Anwendung dieses Modells beschränkte sich meines Wissens neben dem Heidelberger Projekt „Pidgin-Deutsch“, für das es entwickelt wurde, auf die Dissertation Senfts über die Sprache Kaiserslauterer Bergleute (Senft 1982).

Unter den Soziolinguisten, die sich mit der sozialen Bedeutung der sprachlichen Variation beschäftigten, setzten ebenfalls wichtige Lernprozesse ein; insbesondere setzte sich die Einsicht durch, dass die Beziehung zwischen Sprach- und Sozialstruktur nicht im Sinn einer einfachen Korrelation zu fassen ist. Der 1978 von Quasthoff herausgegebene Sammelband „Sprachstruktur - Sozialstruktur“ ging einen wesentlichen Schritt in diese Richtung. In der Einleitung geht Quasthoff nicht (mehr) von einer Widerspiegelung der Sozialstruktur in der Sprachstruktur aus, sondern davon, „daß die ,Sozialstruktur' Bedürfnisse stellt, die mit den sprachlich-kommunikativen Mitteln der "Sprachstruktur“ erfüllt werden“ (1978, S. 8). Sprachliche Strukturen sind nicht fest mit sozialen verbunden, sondern fungieren als „Indexzeichen“. ${ }^{9}$ Das betrifft sogar Anredepronomina, die zu den besten Kandidaten für eine direkte Beziehung zwischen Sprach- und Sozialstruktur gehören:

Typischerweise sind es gerade nicht die realen sozialen Strukturen, sie sich in den sprachlichen Ausdrucksmöglichkeiten und -formen wiederfinden; es sind vielmehr die vorherrschenden ideellen Wertstrukturen einer Gesellschaft, die sich in der sozialen Bedeutung ihrer sprachlichen Systeme widerspiegeln. (Quasthoff 1978, S. 11)

9 Es erscheint plausibel, dass diese Idee auch der Auseinandersetzung mit der Theorie der Interaktionalen Linguistik John Gumperz' geschuldet war, dessen Auffassung von sprachlichen Kontextualisierungsmerkmalen in dem Sammelband durch einen ins Deutsche übersetzten Beitrag vertreten war (Gumperz 1978; vgl. zu Gumperz u.a. Auer/di Luzio (Hg.) 1992; Auer/Roberts 2011 sowie Auer/Heller/Roberts i.Dr.; Auer i.Dr.). 
Erstmals wird auch - unter dem Einfluss der Ethnomethodologie - die Auffassung formuliert, dass die Verwendung bestimmter sprachlicher Mittel soziale Strukturen konstituiert, nicht nur umgekehrt (ebd., S. 13). Langfristig führte dies zu einer konstruktivistischen Soziolinguistik, die die Wirkung sprachlichen Handelns unter Nutzung oder Vermeidung bestimmter sprachstruktureller Merkmale für die Konstruktion der sozialen Wirklichkeit untersucht.

\subsection{Sprachpolitik und Minderheiten}

Bis Mitte der 1970er Jahre ist das Thema Mehrsprachigkeit und Migration abgesehen von der marginalen Kloss-Schule in Deutschland nicht präsent; erst dann kommt es über die ersten Projekte zum Deutsch-Erwerb von Gastarbeitern langsam ins Spiel, allerdings über den Umweg der Untersuchung des ungesteuerten Zweitspracherwerbs von Arbeitsimmigranten. Im „Heidelberger Projekt Pidgindeutsch“ wurde gezeigt, dass der erfolgreiche Erwerb des Deutschen als Zweitsprache durch ,Gastarbeiter von deren sozialer Integration (und dem Einreisealter) abhängt (vgl. Heidelberger Projekt Pidgindeutsch 1975; Becker/Dittmar/ Klein 1978). Die im Rahmen dieses Projekts vorgetragene These vom PidginCharakter des Gastarbeiterdeutsch führte zu einer vehementen Auseinandersetzung mit dem Romanisten Meisel (Meisel 1975). Als Folge dieser Auseinandersetzung wurde auch das Thema Pidgins und Kreolsprachen zumindest kurzfristig für die germanistische Soziolinguistik relevant. Die Phase der deutschen Soziolinguistik, in der das Thema Mehrsprachigkeit beherrschend wurde, beginnt jedoch erst deutlich später.

\section{Die deutsche Soziolinguistik 1980-2000}

Die 1980er und 1990er Jahre sind die Zeit, in der sich die deutsche Soziolinguistik konsolidiert und professionalisiert. Aus den gesellschaftlichen Entwicklungen ergaben sich neue Themen wie die feministische Linguistik sowie die Forschung zur Sprachentwicklung in den neuen Bundesländern, dafür verschwindet das Thema soziale Sprachbarrieren in der Schule, wenn man von einigen Nachzüglerarbeiten (Rosenberg 1986) sowie dem Versuch einer Wiederbelebung durch Ammon/Kellermeier (1997) absieht. 


\subsection{Sprachwandel}

Ab den 1980er Jahren lassen sich neue Impulse in der Soziolinguistik konstatieren, die ihre Wurzeln in der Dialektologie haben; neben einer weiter starken konservativen Richtung, die sich mit der Dokumentation der Grunddialekte (insbesondere im Format von Dialektatlanten und -wörterbüchern) beschäftigte, wandten sich einige germanistische Linguisten der Beschreibung der Variation zwischen Dialekt und Standard zu, insbesondere der Erforschung von Regionaldialekten (vgl. z.B. Mihm 2000) und der Demotisierung (Mattheier 1997) des Standards, die mit der Herausbildung von regionalen Standards einhergeht. Ein singulärer Beitrag zum ersten Themenbereich ist Bellmanns zweidimensionaler Atlas des Mittelrheingebiets, der leider in der Atlasdialektologie ohne größere Nachfolgeprojekte geblieben ist (Bellmann (Hg.) 1994-2002). Er zeigt durch den Vergleich zweier Kartentypen mit traditionellen und moderneren Sprechern Bewegungen im Raum, die auf sozial gesteuerten Wandel (in Richtung auf eine Annäherung an großflächigere, nicht unbedingt immer standardnähere Formen eines Regionaldialekts) hindeuten.

Eine wichtige Rolle spielten in den 1980er Jahren die sozio-dialektologischen Arbeiten Mattheiers, die einerseits an ältere germanistische Traditionen anknüpften, andererseits der Variationsforschung neue Wege wiesen, wie nicht zuletzt die zahlreichen Dissertationsprojekte unter Mattheiers Leitung in dem einflussreichen Heidelberger Graduiertenkolleg 117 „Dynamik von Substandardvarietäten“ (1997-2002) belegen. Mattheiers Verdienst ist es auch, die historische Soziolinguistik neu belebt zu haben. Er ging den von Besch in seiner Freiburger Habilitationsschrift (1967) eingeschlagenen Weg zur Rekonstruktion der sozialen Bedingungen weiter, die die Herausbildung der deutschen Standardsprache ab 1500 gesteuert haben. Am Beispiel von Köln zeigt er (vgl. z.B. Mattheier 1981) die Konkurrenz zwischen oberdeutschem und mitteldeutschem Standard auf und zeichnet die verschiedenen Phasen der Überschichtung der Stadtsprache nach. Dabei wirken die verschieden konturierten Prestiges dieser Varietäten; die ,katholische‘, oberdeutsch-kaiserliche Hochsprache hat im katholischen Köln bis zu Beginn des 19. Jahrhundert die Oberhand. Gesellschaftliche Position und Bildung des Schreibers bestimmen seinen Schreibstil. Mattheiers Konzept der „Leitvariablen“ bringt das Konzept der Salienz ins Spiel. In derselben thematischen Forschungstradition stehen Mihms (und später Elmentalers) Arbeiten zur Übernahme der neuen Standardvarietäten (zunächst über Köln vermittelt) im Rhein-MaasRaum (z.B. Elmentaler et al. 2000).

Besch und Mattheier gebührt auch der Verdienst, im so genannten Erp-Projekt (so die Bezeichnung eines ehemaligen Dorfs im ,Weichbereich“ von Köln, das in der Zeit Teil der Verwaltungseinheit Erftstadt wurde) erstmals in der neueren 
Soziolinguistik die Rolle der Pendler und entsprechend die Umgestaltungen des (ehemals) ländlichen Sprachraums im Einzugsbereich der Großstädte untersucht zu haben (Mattheier 1980a; vgl. dazu inzwischen auch Britain 2012). Dabei wurde die „private“ und die „öffentliche“ Sprache der Informanten verglichen, d.h. es erfolgte eine Analyse sozialer Stile.

Typisch für Mattheiers Vorgehen ist die Kombination von Sprachbeschreibung, Sprachgebrauchsanalyse und Analyse des „Sprachwertsystems“. Mattheier entwickelte schon in den 1980er Jahren, lang vor der Rezeption der Prestonschen folk linguistics, eine „Dialektologie des Sprechers“, in die ältere Ansätze von Mitzka (siehe oben) und Büld (1939) einflossen. Die „Sprecherurteile“ spielten entsprechend im Erp-Projekt eine große Rolle (vgl. Hufschmidt et al. 1983).

Die Analyse des sprachlichen Verhaltens erfolgte im Erp-Projekt mit quantitativen Methoden (vgl. z.B. Lausberg 1993). Auffällig ist aber, dass keine Variablenregeln verwendet wurden. Der Grund ist einfach: Die Labovschen Variablenregeln können nur auf Variation innerhalb einer Varietät angewendet werden, nicht jedoch auf die Dynamik zwischen zwei Varietäten, etwa zwischen Dialekt und Standard. So ist die von Schlobinski (1987) im Format einer Variablenregel untersuchte silbenanlautende $g$-Schwächungsregel im Berlinischen zwar ein aus dem Niederdeutschen über Substrateinfluss entlehnter Prozess, sie operiert jedoch im Berlinischen quasi ,lautgesetzlich` und wird durch Faktoren des sprachlichen Kontexts ebenso wie durch soziale und situative Faktoren in ihrer Anwendung lediglich gefördert oder behindert. Es gibt im deutschen Sprachraum auch andere Variablen dieser Art, etwa die ch-Koronalisierung (vgl. Herrgen 1986). Anders verhält es sich aber mit Dialekt/Standard-Variablen, die auf historisch bestimmten lexikalischen Klassen operieren. So konnte die Berlinische Variation zwischen /e:/ und std. /ai/ wie in weeßte, been von Schlobinski nicht auf dieselbe Weise analysiert werden wie die $g$-Erweichung, denn es gehen ja keineswegs alle std. /ai/ in berlinisch /e:/ über (oder gar umgekehrt), sondern lediglich die Reflexe der mhd. Diphthonge; die Wahrscheinlichkeit der monophthongischen Realisierung ist überdies von Lexem zu Lexem verschieden. Gerade für die sprachliche Situation im mittel- und oberdeutschen Raum, wo solche Variationsphänomene eine große Rolle spielen, mussten daher andere Modelle entwickelt werden, die mit lexikalischen Korrespondenzregeln (input switch rules) zwischen Dialekt und Standard operieren. Wegweisend dafür waren die Arbeiten Dresslers zum Wienerischen. Bidialektale Modelle tragen der Standard/DialektDynamik in Deutschland, Österreich und vielen anderen europäischen Ländern weit besser Rechnung als das für die nordamerikanische Situation wohl adäquatere Modell der Variablenregel (vgl. Dressler/Wodak 1982; Rennison 1981; Moosmüller 1987; Auer 1990, 1993). 
Die besprochenen Arbeiten machen bereits deutlich, dass die 1980er und 1990er Jahre auch die große Zeit der deutschen Stadtsprachenforschung sind (vgl. dazu auch u.a. Auer 1990 zu Konstanz; Brinkmann to Broxten 1986 zu Frankfurt; Steiner 1994 zu Mainz; Salewski 1998 zu Duisburg).

\subsection{Soziale Gruppen und ihre Sprache}

Das größte Stadtsprachenprojekt, das jemals in Deutschland durchgeführt wurde, war das im IDS von Werner Kallmeyer Mitte der 1980er bis Mitte der 1990er Jahre geleitete Projekt „Kommunikation in der Stadt“ (vgl. Kallmeyer (Hg.) 1994-1995). Dieses Projekt hatte nicht die Absicht, Sprachwandel zu untersuchen (wie die meisten im letzten Abschnitt genannten Arbeiten zur Variabilität der Stadtsprache); vielmehr war es sein Ziel, mittels ethnografischer und geprächsanalytischer Erfassung des Sprachverhaltens in mehreren Mannheimer Stadtvierteln mit unterschiedlicher sozialer Zusammensetzung Erkenntnisse darüber zu gewinnen, wie sprachliche Variation in der Interaktion eingesetzt wird, um soziale Zugehörigkeit zu signalisieren. Das Projekt stand damit in einer völlig anderen Tradition als die bisher genannten und griff u.a. auf den Begriff „soziale Welten“, wie er in der Chicagoer Schule des Symbolischen Interaktionismus in der Soziologie entwickelt worden war, zurück, später auch auf Gumperz' Interaktionale Soziolinguistik.

Gumperz, der in den 1980er Jahren mehrfach als Gastprofessor zunächst an der Universität Konstanz, später auch am IDS tätig war, nahm in dieser Phase einen nachhaltigen Einfluss auf die deutsche Soziolinguistik. Er selbst hatte seine noch strukturalistisch geprägte Dissertation an der University of Michigan über ein germanistisches, dialektologisches Thema (schwäbische Diaspora-Dialekte) geschrieben und kannte sich in der deutschen Vorkriegsdialektologie gut aus. Seine anthropologische Arbeit in Indien hatte ihn aber zu der Überzeugung gebracht, dass ein völlig neuer Ansatz notwendig war, um die Verbindung von sozialen und sprachlichen Strukturen zu erfassen (vgl. Auer i.Dr.). Ausgangspunkt ist für ihn in seiner späten Phase nicht mehr das Sprachsystem einer einzelnen Varietät, sondern die Interaktion, in der der Sprecher oder die Sprecherin Elemente ihres multidialektalen und/oder multilingualen sprachlichen Repertoires aktiviert, um auf diese Weise Äußerungen in der einen oder anderen Weise $\mathrm{zu}$ „kontextualisieren“ und so soziale und interaktionale Bedeutungen zu generieren. Damit treten zum einen die Sprecher/innen in den Vordergrund, die ihre sprachlichen Ressourcen situationsspezifisch mit verschiedenen Intentionen einsetzten (vgl. ähnlich auch Macha 1991). Zum anderen muss die Struktur und sequenzielle Entwicklung der Interaktion mit in die Analyse einbezogen werden: Sprecher wechseln ihre sprachlichen Mittel nach Gumperz’ Auffassung, um die 
Rahmung der Interaktion zu verändern, so wie bilinguale Sprecher den Code wechseln. Diese Erkenntnis führte zur Integration konversationsanalytischer Methoden in die Soziolinguistik (vgl. Auer 1984, 1986, 1990, S. 211-218; vgl. Gumperz 1994). Gumperz selbst sah seinen Ansatz der Zeichentheorie Peirce' - und nicht der Saussures - verpflichtet. Seine Methode kombiniert ethnografische und konversationsanalytische Verfahren. Dies setzt zwar ein profundes Wissen über die den Sprechern zur Verfügung stehenden sprachlichen Ressourcen voraus, zugleich entfällt aber das Primat des Systems. In Deutschland leitete das Mannheimer Stadtspracheprojekt den Übergang zu einer Soziolinguistik der indexikalischen Systeme ein, wie sie nach der Jahrtausendwende von Eckert und Silverstein propagiert wurde und inzwischen weltweit tonangebend ist.

Die beiden großen gesellschaftlich-politischen Herausforderungen, auf die die Soziolinguistik in dieser Phase reagieren musste, waren die veränderten Gender-Rollen und der Zusammenbruch der DDR. In beiden Fällen zeigt sich an der Art und Weise, in der die deutsche Soziolinguistik sich des Themas annahm, der Konflikt zwischen der traditionelleren korrelativen und der neueren indexikalisch-konstruktivistischen Soziolinguistik.

Die so genannte feministische Linguistik war zu großen Teilen keine empirische, sondern eine normative Bewegung, die die Veränderung des Sprachgebrauchs von oben zum Ziel hatte und damit zumindest auch teilweise erfolgreich war. Es gab aber auch eine empirische Gender-Forschung, die mit quantitativkorrelativen Versuchen begann (,Männer sprechen anders als Frauen'). Diese Untersuchungen hatten insbesondere das Diskursverhalten im Auge (Trömel-Plötz 1982). ${ }^{10}$ Der Ansatz erwies sich als wenig ertragreich und wurde zunehmend von Untersuchungen ersetzt, die bei schärfer konturierten Gruppen oder communities of practice ansetzten und die Rolle der Sprache für die Konstitution dieser Gruppen nicht zuletzt mit ethnografischen Methoden thematisierten. Eine der ersten Arbeiten in dieser Tradition war Streecks im Rahmen des Mannheimer Stadtspracheprojekts entstandene Arbeit über eine Gruppe älterer Mannheimer Unterschichtsfrauen, die durch sexuelle Witze ihre Erfahrungen eben in ihrer Rolle als ältere Unterschichtsfrauen bearbeiten. Untersucht wird ,ihre Weise, sich gemeinsam mit ihrem Leben auseinanderzusetzen“ (Streeck 1988, S. 54), wozu auch die Selbst- und Fremdkategorisierung gehört. Diese Tradition ethnografischer und interaktionslinguistischer, genderbezogener Forschung in Gruppen setzt sich bis in die Gegenwart fort (vgl. z.B. Spreckels 2006 und verschiedene Beiträge in Kotthoff/Mertzlufft (Hg.) 2014). Die feministische Soziolinguistik rückte dabei

10 Zur Relevanz von Gender im phonologischen und grammatischen Sprachwandel vgl. Kotthoff (1992). 
immer mehr von einem essentialistischen Gender-Begriff ab und begriff Gender zunehmend als Performanzphänomen (im Sinne Judith Butlers). Es geht um doing gender, nicht mehr um being gender (vgl. Kotthoff 2002 für einen Überblick über die Diskussionslage zur Jahrtausendwende).

Die Wiedervereinigung führte ebenfalls zu einer weit ausdifferenzierten soziolinguistischen Forschung (vgl. den Überblick bei Stevenson 2002). Sie reichte von variationslinguistischen Arbeiten insbesondere zu den beiden Teilen Berlins und der Rolle der Berliner Umgangssprache im Berliner Osten (vgl. dazu bereits vor der Wende Schlobinski 1987; ebenfalls Schlobinski 1996; Reiher 2001; Dittmar 2000) über Forschungen zur Ost-West-Migration aus Sachsen in westliche Bundesländer (Barden/Großkopf 1998; Auer/Barden/Großkopf 2000) und zur Auswirkung der Staatsgrenze auf Dialektgrenzen (Harnisch 2010) bis zu diskursanalytischen Rekonstruktionen des Mauerfalls (Dittmar 1997; Bredel 1999) und zu Untersuchungen zur Aneignung westlicher Gattungen wie des Bewerbungsgesprächs durch ostdeutsche Sprecher in der Nachwendezeit (Birkner 2001; Kern 2000).

In dieselbe Zeit fällt auch der Beginn der allerdings in Deutschland nur zögerlich entwickelten interkulturellen Interaktionsanalyse, der ebenfalls von John Gumperz beeinflusst war (Kotthoff 1991; Günthner 1991). Das Modell der interkulturellen Kommunikation wurde übrigens auf beide hier erwähnte Themen angewendet, also sowohl auf die Interaktion zwischen Ost- und Westdeutschen (Auer/ Kern 2001) als auch auf die Interaktion zwischen Männern und Frauen (in der Tradition von Deborah Tannen; vgl. Günthner 1992).

Die Frage des Zusammenhangs zwischen sozialen Schichten und Sprache spielte in dieser Phase der Soziolinguistik kaum mehr eine Rolle. Bemerkenswert ist auch, dass die Rezeption Bourdieus sehr schwach bleibt (seine Theorien fanden fast nur in der Erziehungswissenschaft ein Echo, vgl. Gogolin 1992). Es herrschte eine grundsätzliche Skepsis sozialen Schichtenmodellen gegenüber. Dies entsprach wohl der Auffassung von der Bundesrepublik als einer sozial wenig differenzierten Gesellschaft.

\section{Entwicklungen seit der Jahrtausendwende und die Soziolinguistik der Zukunft}

Um die Jahrtausendwende lässt noch einmal einen Einschnitt in der Entwicklung der Soziolinguistik ansetzen. Seine Rechtfertigung ergibt sich vor allem aus der Art und Weise, wie seither das Thema Migration und Mehrsprachigkeit in den Vordergrund getreten ist. Fast nicht davon zu trennen ist das Interesse für jugendliche Sprechweisen, das wiederum an die Untersuchung der Sprachverwendung 
in den neuen Medien gekoppelt ist. In Kurzform zusammengefasst, scheint mir die Soziolinguistik seit der Jahrtausendwende durch die folgenden Entwicklungen charakterisiert zu sein:

a) In der Sozio-Dialektologie geht das Interesse für die Struktur und Entwicklung des Variationsbereichs zwischen Grunddialekt und regional ungebundener Standardvarietät weiter (vgl. u.a. Auer 2005; Auer/Spiekermann 2011; Schmidt/Herrgen 2011; Auer/Schmidt (Hg.) 2010; Spiekermann 2008; Lenz 2003). Die korpuslinguistischen Verfahren sowie die kartenorientierten Darstellungsformen profitieren dabei von neuen statistischen Methoden (regressionsstatistische Modelle, multidimensional scaling, Cluster- und Interpolationsverfahren; vgl. in Deutschland z.B. Kehrein 2012; Schwarz erscheint). Dabei werden aber keine grundsätzlich neuen soziolinguistischen Erklärungsmodelle verwendet. Der früher dominante Faktor der sozialen Schicht bleibt als Erklärung für sprachliche Unterschiede weiter marginal (oder wird nur in der historischen Soziolinguistik diskutiert, z.B. in Bezug auf die Entwicklung der Arbeitersprache im 19. Jahrhundert; vgl. Mihm 1998; Elspaß 2005). Tatsächlich scheint sich die Sicht auf die Beziehung zwischen Dialektverwendung und sozialer Schicht inzwischen grundlegend verändert zu haben. Dialekt wird keineswegs mehr als Bildungsbarriere gesehen und auch nicht mehr als Merkmal eines bildungsfernen Sprachstils stigmatisiert; vielmehr ist der Diskurs über Dialektkompetenz dadurch geprägt, dass dem Dialekt/Standard-Sprecher dieselben positiven neuro-kognitiven Vorteile bescheinigt werden wie klassischen Bilingualen.

b) Das Interesse für die Perspektive der Sprecher auf Sprache (Einstellungen und Wissen, insbesondere über geografische Verteilung) hat nach der Jahrtausendwende unter dem Einfluss der Arbeiten Prestons (Preston 1989 u.a.) auch in der germanistischen Soziolinguistik zugenommen (vgl. u.a. Anders/Hundt/Lasch (Hg.) 2010; Stöckle i.Dr.). Die Bedeutung von Einstellungen für den Sprachwandel ist schon seit langem bekannt. Zusätzlich wird jedoch inzwischen auch die Struktur des Laienwissens über sprachliche Varianten als eine wichtige Determinante sprachlicher Veränderungen verstanden. Allerdings steht der Beweis noch aus, dass zwischen ethnodialektologischem Wissen und Sprachwandel tatsächlich ein kausaler Zusammenhang besteht.

c) Das Interesse der Sprachwandelforschung hat sich von eher unbewussten auf bewusste, oft stereotypisierte Merkmale verlagert. Hand in Hand damit geht eine gewisse Präferenz für die Untersuchung performativer Strategien, mittels derer Sprecher und Sprecherinnen sich sozial verorten. Besonders jugendliche Sprechweisen werden als bricolage aus Merkmalen verschiedenster Provenienz gesehen, deren indexikalische Bedeutung sich schnell verändern kann (vgl. Androutsopoulos 1998; Androutsopoulos/Georgakopolou (Hg.) 2003; Schlobinski/Heins (Hg.) 1998). Vor allem mediale Selbstinszenierungen in den neuen Medien (SMS, 
Chats, Internet-Auftritte) sind inzwischen in einer Vielzahl von Arbeiten untersucht worden. Es gibt allerdings kaum Arbeiten, die mediales und face-to-faceVerhalten anhand derselben Sprecher aufeinander beziehen und mögliche Unterschiede herausarbeiten.

d) Seit der Jahrtausendwende ist das soziolinguistische Interesse an der Sprache von Jugendlichen mit Migrationshintergrund enorm gewachsen. Nach einer langen Phase, in der Mehrsprachigkeit hauptsächlich im Zusammenhang des Zweitspracherwerbs (oder bilingualen Erstspracherwerbs) aus psycholinguistischer Perspektive untersucht wurde (aber: Auer 1984; Auer (Hg.) 1998), wird seither auch das mehrsprachige Verhalten selbst und seine soziale Bedeutung stark thematisiert. Keim zeigt (z.B. 2007) den Wandel bilingualer Praktiken in einer Gruppe jugendlicher Mädchen mit türkischem Familienhintergrund. Dirim/Auer (2004) konnten anhand einer ethnografischen Untersuchung in Hamburg belegen, dass sich in multiethnischen Gruppen bilinguale Sprechstile entwickeln, zu der die Verwendung von Fragmenten anderer Migrationssprachen als der ,eigenen` (insbesondere des Türkischen) gehören.

$\mathrm{Zu}$ den untersuchten jugendlichen Stilen gehören auch neue, poly-ethnische Formen oder zumindest Stile des Deutschen (Multi-Ethnolekte). Diese und ihre Transformationen in den Medien sowie in der Verwendung durch monolinguale und monoethnische deutsche Jugendliche (crossing) hat auch in Deutschland zu vehementen soziolinguistischen Debatten geführt (vgl. u.a. Androutsopoulos 2007; Auer 2003; Auer 2013a; Clyne 2000; Deppermann 2007; Freywald et al. 2011; Siegel 2014; Wiese 2006, 2012), die sich auf die Bewertung dieser Sprechweisen, ihren Status (als neue Varietät oder sogar Dialekt des Deutschen), ihre soziale Verbreitung und ihre Bedeutung für einen möglichen Sprachwandel im Deutschen insgesamt beziehen. Allerdings fehlen für die Beantwortung mancher dieser Fragen immer noch empirisch ausdifferenzierte, verlässliche Untersuchungen. e) Schon seit der Mitte der 1990er Jahre gibt es eine bemerkenswerte Revitalisierung der Forschung zur Form und Verwendung des Standarddeutschen in den Ländern, in denen es Amtssprache ist, aber auch zu Gesellschaften, in denen Minderheiten deutsch sprechen oder sprachen oder wo Deutsch als Fremdsprache verwendet wird. Die Gründe dafür mögen teils ganz praktisch in den heute deutlich besseren Forschungsmöglichkeiten in Osteuropa liegen; auch die nach 1989 neu definierte Bedeutung des wirtschaftlich und politisch nun gewichtigeren Deutschlands in Europa und in einer globalisierten Welt - und der damit verbundenen Frage nach dem Stellenwert des Deutschen in Europa und in der Welt wird wohl eine Rolle gespielt haben. Mit dem Ende der deutschen Doppelstaatlichkeit scheinen auch gewisse soziolinguistische Tabus verschwunden zu sein.

Die Diskussion über den polyzentrischen Charakter des Deutschen greift unmittelbar auf Überlegungen Kloss’ zurück. Sie begann bereits Mitte der 1990er 
Jahre mit Beiträgen von Clyne (1995) und Ammon (1995) und scheint anzudauern. Die Entwicklung verschiedener Varietäten des Standarddeutschen in Österreich und der deutschsprachigen Schweiz ist allerdings weniger eine Frage der tatsächlichen sprachlichen Divergenz als eine der sprachideologischen Enkodierung von Differenzen (Auer 2013b), die selbst einer eingehenden soziolinguistischen Untersuchung bedarf.

Die ebenfalls an Kloss erinnernde Frage, ob das Deutsche weltweit gesehen an Bedeutung zu- oder abnimmt, ist ebenfalls wieder aktuell. Aus der Anzahl der Sprecher des Deutschen in Europa werden Ansprüche auf die Verwendung der Sprache z.B. in den europäischen Institutionen abgeleitet. Die Bedeutung des Deutschen als Wissenschaftssprache und die Dominanz des Englischen wird diskutiert (vgl. Ammon 1998). Forschungen zu den deutschsprachigen Minderheiten v.a. in Osteuropa sind wieder en vogue (Rosenberg 1994 etc.; Gawrisch/Ammon 2010; Eichinger/Plewnia (Hg.) 2008); und selbst die sprachlich relativ wenig einflussreiche deutsche Kolonialzeit ist zum Forschungsthema geworden (vgl. u.a. Stolz (Hg.) 2011).

f) Die am Anfang der deutschen Soziolinguistik stehende Sprachbarrierendiskussion feierte in der Zeit nach der Jahrtausendwende eine erstaunliche Renaissance, wenn auch in einem neuen Gewand. Dem Schock der 1960er Jahre, als Picht die Bildungskatastrophe verkündete, entsprach nun der Schock der ersten PISA-Studie (siehe Baumert et al. (Hg.) 2001). Wie damals setzte sich die politische Erkenntnis durch, dass es sich der Wirtschaftsstandort Deutschland nicht erlauben kann, einen großen Teil seiner Schüler mit ungenügenden Lese- und Schreibkenntnissen aus der Schule zu entlassen und damit ihre beruflichen Karrieren zu belasten. Die Reproduktion sozialer Ungleichheit durch die Schule wird nun aber völlig ihrer schichtensoziologischen Begründung und Einbettung entkleidet und ausschließlich in Bezug auf den Faktor Migrationshintergrund diskutiert. Damit erscheint die Mehrsprachigkeit selbst als Problem (vgl. die Beiträge in Gogolin/Neumann (Hg.) 2009 zum so genannten Esser-Report). Eine jüngere Untersuchung von Steinig et al. (2009), die die schriftlichen Leistungen (Filmnacherzählungen) von Viertklässlern im Jahr 1972 und im Jahr 2002 vergleicht, kommt jedoch zu dem Ergebnis, dass die soziale Selektion durch die Schule heute wesentlich besser von den schriftsprachlichen Leistungen der Schüler vorhergesagt wird als vor 40 Jahren, und dass dieses Ergebnis nur teilweise auf den Migrationshintergrund der Schüler zurückgeführt werden kann (ebd., S. 345). ${ }^{11}$ Die Autoren kommen zu der Schlussfolgerung:

11 Diskriminanzanalysen weisen nach wie vor den Merkmalen „Kasusmarkierung“ (Ruhrgebiet) und Rechtschreibung einen wichtigen Einfluss zu; 2002 ist allerdings der Wortschatz der beste 
Es erscheint uns geradezu als Ironie soziolinguistischer Forschung, dass in unserer Zeit, in der die Theorie Bernsteins nur noch bruchstückhaft bekannt ist, seine Annahmen eine vergleichsweise hohe Erklärungskraft haben, während sie in den 1970er Jahren in der (sic) seine Thesen in der Soziolinguistik wie in der Sprachdidaktik intensiv diskutiert wurden, in der Bundesrepublik empirisch nicht bestätigt werden konnten, jedenfalls nicht überzeugend. (Steinig et al. 2009, S. 353)

Die Autoren beklagen weiterhin, dass die Diskussion sich „seit Ende der 1970er Jahre“ immer mehr der Zweisprachigkeit zugewandt hat; dabei korreliere der Faktor Schicht aber mit den meisten untersuchten sprachlichen Merkmalen wesentlich besser als der Faktor Zweisprachigkeit (ebd., S. 369). Bemerkenswert ist vielleicht auch, dass das, was in den 1970er Jahren als kompensatorische Spracherziehung höchst kritisch diskutiert wurde, heute als frühkindliche und vorschulische Sprachförderung (im Deutschen!) mit großer Selbstverständlichkeit als Königsweg zur Verbesserung der schulischen Leistungen von Kindern mit Migrationshintergrund gesehen wird.

\section{Abschließende Bemerkungen}

In den letzten 50 Jahren ist in der Soziolinguistik in Deutschland viel passiert. Wie in allen Teilbereichen der Linguistik waren die frühen Jahre der sich selbst erfindenden Soziolinguistik bis etwa 1970 von einer Mischung aus Aufbruchsstimmung und heute teils naiv anmutendem Import von Modellen aus dem Ausland gekennzeichnet (in diesem Fall zunächst aus England). Inzwischen hat sich die Soziolinguistik längst professionalisiert, diversifiziert und dabei theoretisch und methodisch weiterentwickelt. Nicht ganz ohne Neid konstatiert man allerdings, dass die (Sozio-)Linguistik während der späten 1960er und 1970er Jahren im Zentrum der gesellschaftlichen Diskurse agierte. Wir sind heute besser, aber auch bescheidener, und wir werden viel weniger gehört. Das liegt teils an uns, teils an den gesellschaftlichen Entwicklungen.

Dabei gibt es viel zu tun. Wir sprechen heute anders als vor 50 Jahren. Zu den wichtigsten soziolinguistischen Themen gehören die massive Umstrukturierung

\footnotetext{
Prädiktor; Schriftbild, Verbmorphologie (vermutlich durch den Einfluss der Migration) und Anteil der Hypotaxe spielen ebenfalls eine Rolle (Steinig et al. 2009, S. 350). Die Unterschiede zwischen den älteren und den neueren Daten sind teils positiv, teils negativ zu bewerten. Unter den Kindern mit Gymnasialempfehlung gebe es heute eine stärkere Fähigkeit zur „variantenreicheren erzählerischen Textgestaltung “; besonders bei den Kindern aus der Unterschicht haben hingegen die Rechtschreibleistungen abgenommen (ebd., S. 345).
} 
der Beziehung von Mündlichkeit und Schriftlichkeit, die riesige Anzahl von nichtmuttersprachlichen, plurilingualen Sprechern und Sprecherinnen des Deutschen in Deutschland und die grundsätzliche Mehrsprachigkeit unserer Schulen, die Auswirkungen der immer noch vergleichsweise bescheidenen, aber sich doch entwickelnden deutsch-englischen Zweisprachigkeit, die radikale Umgestaltung des Gefüges aus Dialekt und Standard innerhalb der letzten 50 Jahre und die neue Bedeutung dialektaler Sprechweisen für die regionale und lokale Identitätsdarstellung in einer globalisierten Welt, das Verschwinden alter Leitmedien (Buch, Bühne) und die zentrale Bedeutung neuer schriftlicher Medien. Die Themen sollten der Soziolinguistik also auch in Zukunft nicht ausgehen.

\section{Literatur}

Ammon, Ulrich (1973a): Dialekt, soziale Ungleichheit und Schule. 2., überarb. Aufl. Weinheim.

Ammon, Ulrich (1973b): Dialekt und Einheitssprache in ihrer sozialen Verflechtung: eine empirische Untersuchung zu einem vernachlässigten Aspekt von Sprache und sozialer Ungleichheit. (= Pragmalinguistik 3). Weinheim.

Ammon, Ulrich (1995): Die deutsche Sprache in Deutschland, Österreich und der Schweiz. Das Problem der nationalen Varietäten. Berlin/New York.

Ammon, Ulrich (1998): Ist Deutsch noch internationale Wissenschaftssprache? Englisch auch für die Lehre an den deutschsprachigen Hochschulen. Berlin/New York.

Ammon, Ulrich/Kellermeier, Birte (1997): Dialekt als Sprachbarriere passé? 25 Jahre danach. Versuch eines Diskussions-Erweckungsküsschens. In: Deutsche Sprache 25, S. 21-38.

Anders, Christina Ada/Hundt, Markus/Lasch, Alexander (Hg.) (2010): Perceptual dialectology. Neue Wege der Dialektologie. (= Linguistik - Impulse \& Tendenzen 28). Berlin/New York.

Androutsopoulos, Jannis (1998): Deutsche Jugendsprache: Untersuchungen zu ihren Strukturen und Funktionen. (= VarioLingua 6). Frankfurt a.M. u.a.

Androutsopoulos, Jannis (2007): Ethnolekte in der Mediengesellschaft. Stilisierung und Sprachideologie in Performance, Fiktion und Metasprachdiskurs. In: Fandrych, Christian/ Salverda, Reinier (Hg.): Standard, Variation und Sprachwandel in germanischen Sprachen. (= Studien zur Deutschen Sprache 41). Tübingen, S. 113-157.

Androutsopoulos, Jannis/Georgakopoulou, Alexandra (Hg.) (2003): Discourse constructions of youth identities. (= Pragmatics \& Beyond, N.S. 110). Amsterdam, S. 197-219.

Auer, Peter (1984): Bilingual conversation. (= Pragmatics \& Beyond 5, 8). Amsterdam.

Auer, Peter (1986): Konversationelle Standard/Dialekt-Kontinua (Code-Shifting). In: Deutsche Sprache 14, S. 97-124.

Auer, Peter (1990): Phonologie der Altagssprache. Eine Untersuchung zur Standard/Dialekt-Variation am Beispiel der Konstanzer Stadtsprache. (= Studia linguistica Germanica 28). Berlin u.a.

Auer, Peter (1993): Bidimensionale Modelle für die Beschreibung von Standard/ DialektVariation und ihre Vorläufer in der deutschen Dialektologie. Ein Beitrag zur historischen Kontinuität. In: Viereck, Wolfgang (Hg.): Verhandlungen des Internationalen 